Review Article

\section{Biologic therapy in severe asthma: An}

\section{update}

\author{
Maha A Al Ammari* \\ Pharmaceutical Care Department, Ministry of the National Guard - Health Affairs, King Abdullah \\ International Medical Research Center, King Saud bin Abdulaziz University for Health Sciences, \\ Riyadh, Saudi Arabia
}

\section{Abstract}

Asthma is a chronic inflammatory disease of the airways characterized by airway inflammation, bronchial hyperresponsiveness, reversible airflow obstruction and recurrent symptoms. Patients often present with coughing, wheezing, dyspnea, and chest tightness, were they usually responds to the mainstay of treatment that relies on inhaled glucocorticoids (ICS), and long acting $\beta 2$ agonist (LABA), along with leukotriene. In around $20 \%$ of the patient's morbidity, mortality and cost of therapy increased because they fail to benefit from the existing gold standard therapy regimen. Both immunoglobulin-E (IgE), interlukin-5 (IL-5) had proven to play important major role in asthma pathogenesis. Over the past two decades biologic therapy that targeting IgE begins the era in treating severe asthma, and recently anti-IL-5, revealed major role in eosinophils maturation, activation, survival, and recruitment process of severe asthma. The different biologic therapy that is currently available in the market are supported by solid evidence from controlled randomized clinical trials, to guide the clinician on the type of patients that will benefit from the therapy, with an insight on the appropriate monitoring parameters and patient evaluation plans. This review was conducted by searching PubMed, EMBASE, and Google Scholar to identify peerreviewed clinical trials, guidelines, and review articles published in English in the role of biologic therapy in severe asthma. The main aim from publishing this review is to summarize the current available evidence on the approved biologic therapy in treating patients with severe asthma.

\section{More Information}

*Address for Correspondence: Maha A Al Ammari, MSc, MPH, Pharmaceutical Care Department, Ministry of the National Guard, Health Affairs, King Abdullah International Medical Research Center, King Saud bin Abdulaziz University for Health Sciences, Riyadh, Saudi Arabia, P.O. Box 22490, Tel: +966-18011111 Ext. 18731; +966506409887; Email: ammarima4@gmail.com; ammarima@ngha.med.sa

Submitted: 20 August 2019 Approved: 27 August 2019 Published: 28 August 2019

How to cite this article: Al Ammari MA. Biologic therapy in severe asthma: An update. Arch Asthma Allergy Immunol. 2019; 3: 003-009. DOI: dx.doi.org/10.29328/journal.aaai.1001016 ORCID: orcid.org/0000-0001-9105-9996 Copyright: @ 2019 Al Ammari MA. This is an open access article distributed under the Creative Commons Attribution License, which permits unrestricted use, distribution, and reproduction in any medium, provided the original work is properly cited

Keywords: Biologic therapy; Severe asthma; Omalizumab; Mepolizumab; Benralizumab; Reslizumab

Check for updates

\section{Introduction}

Asthma is defined as a heterogeneous disease, usually characterized by chronic airway inflammation. It is characterised by a history of respiratory symptoms such as wheezing, dyspnea, chest tightness and cough varying over time and intensity, as well as expiratory airflow limitation [1]. Asthma symptoms are either episodic or persistent, affecting up to $18 \%$ of the Saudi population and prevalent in both adults and children [2-5]. Symptom control in the majority of asthma patients is usually achieved with the gold standard regimen, inhaled corticosteroid (ICS), however approximately $20 \%$ of patients do not achieve control, increasing mortality, morbidity and overall healthcare cost [4].

\section{Asthma pathogenesis}

Allergen/antigen presentation by antigen presenting cells to Th0 cells cause Th2 cell differentiation, resulting in the production of IL-4, IL-5, and IL-13 cytokines and the activation of B cells to secrete immunoglobulin E [3]. Different chemical mediators induce smooth muscle bronchoconstriction and stimulate the inflammatory response. Histamine, prostaglandins, and leukotrienes are released as a result of the degranulation of mast cell bound IgE. IL-5 also acts as a potent regulator for eosinophil proliferation, differentiation, and activation [3,5-7]. Asthma is classified in four categories based on the immune cells involved in the mechanism. The first category is Type 2 eosinophilic inflammation, which is common in at least $60 \%$ of asthmatic patients. Patients will have a blood eosinophilia level of $\geq 300 / \mu \mathrm{l}$ and a sputum eosinophilia level of $\geq 2 \%$ leukocytes in the sample. The majority of patients will respond well to ICS. Type 2 eosinophilic inflammation asthma is subdivided in two groups, early onset allergic eosinophilic airway inflammation (extrinsic asthma), and late onset nonallergic eosinophilic airway inflammation (intrinsic asthma). The second category is neutrophilic inflammation, where neutrophils account for $40 \%-60 \%$ of leukocytes in an induced sputum sample. This type of asthma is triggered by infection, irritants, and tobacco smoke and the patients mostly do not respond to ICS. The third category is mixed inflammation, where features from both eosinophilic and neutrophilic inflammation types are present. This type is less common, but more severe and difficult to treat. The fourth category is a paucigranulocytic phenotype with airway limitation caused 
by other mechanisms. It is the least common and patients usually have milder disease [2].

Different drug targets had been discovered leading to the advancement of biologic agents to treat patients who are not responsive to ICS, including IgE, IL-4/13, and IL-5, IL-17, TSLP, in addition to many others being investigated. The next section provides an updated review of the United States' FDA approved biologic treatment for severe asthma.

\section{$\lg E$ antagonist}

Omalizumab: Omalizumab is a recombinant humanized monoclonal antibody that binds free IgE, and prevents its binding to high affinity receptors on the mast cells, basophils, and dendritic cells reducing the cellular responses to an allergen. Omalizumab is approved for patients older than 6 years, diagnosed with severe asthma and treated with a high dose of ICS with a long acting $\beta$ - 2 agonist, and an IgE level within therapeutic range. The dose is calculated based on the weight and IgE levels, and administered subcutaneously every 2-4 weeks $[4,8-11,7,12]$.

Thebenefitofomalizumabasadd-ontherapyinuncontrolled patients with severe persistent asthma and treated with ICS and long acting $\beta-2$ agonist therapy, was investigated in the innovate trial. A randomized, double blind, placebocontrolled trial over 28 weeks resulted in reducing the rate of exacerbation by $26 \%$ ( 0.24 in omalizumab group compared to 0.48 in placebo group, $\mathrm{P}=0.002$ ). Emergency department visits was significantly reduced $(0.24$ in omalizumab group compared to 0.43 in placebo group, $\mathrm{P}=0.038$ ). Both quality of life and lung function test was improved in the omalizumab group compared to placebo, with a comparable incidence rate of adverse events [12].

The experience registry which was a two year observational study, evaluated the effectiveness and safety of omalizumab in patients with uncontrolled persistent asthma. The asthma exacerbation rate, symptoms, use of rescue medication and systemic steroid, were assessed. More than $50 \%$ reduction in symptoms and rescue medication use was evident at 24 months. Among the subgroup analysis, the majority (95.6\%) of the responders showed a significant reduction in severe asthma exacerbation symptoms at 24 months. The mean dose of systemic steroid (prednisolone-equivalent) was markedly decreased from baseline $(16.6 \mathrm{mg})$ to month 24 (5.8mg). No significant adverse events were reported throughout the observation period [13].

In the extra trial, a prospective multicenter randomized, double-blind, placebo-controlled study with omalizumab in severe allergic asthma inadequately controlled with standard therapy, at week 48 , the exacerbation rate was significantly reduced in the omalizumab versus placebo group (0.66 Vs. $0.88, \mathrm{P}=0.006$ ), with a $25 \%$ relative reduction (IRR, 0.75 (95\% CI, 0.61 - 0.92)). In addition, omalizumab increased the time to first asthma exacerbation (HR, 0.74 (95\% CI, 0.60 $0.93) \mathrm{P}=0.008$ ). The mean dose for rescue medication was reduced, with an overall reduction in the symptoms score and improvement in the quality of life (QoL) scores [14].

Safety and tolerability of omalizumab was studied extensively. Rare anaphylactic reactions $(0.1 \%$ of the patients) were documented. It should be noted that due to ${ }_{1}$ cardiovascular and cerebrovascular serious adverse events, clinicians should be attentive when prescribing omalizumab. An observational epidemiological study, the EXCELS trial, was conducted to evaluate the long term effectiveness and safety of omalizumab when used in patients with moderate to severe asthma. A higher rate of cardiovascular and cerebrovascular serious adverse events in patients using omalizumab was reported compared to the non-omalizumab group (13.4 vs. 8.1 per 1000 person years (PY's)), which could be explained by the higher rate of severe bronchial asthma in the omalizumab group [15].

Omalizumab has extensive data on its efficacy in improving both clinical and functional parameters in patients with severe asthma, in addition to its effect on the blood and sputum eosinophil count. Unanswered questions include the appropriate duration of therapy, the possibility of developing predictive biomarkers to aid in selecting appropriate patients to initiate the treatment, monitor progress and cessation of therapy $[11,16]$.

Patients initiated on omalizumab should be monitored for 16 weeks before a decision is made regarding their response (responder or non-responder). This is considered a limitation because about one third of patients are non-responders. From an economic perspective, the resources used to treat non-responders for 16 weeks could be devoted to develop predictive biomarkers to initiate and monitor the treatment. A second challenge is to decide the appropriate duration of therapy in responsive patients as its effect persisted in a number of patients after 4-6 years of treatment. However, several reports suggest that withdrawal of omalizumab therapy after successful treatment of patients with severe asthma and with long history of oral glucocorticoids use, results in severe asthma exacerbation [16].

\section{IL-5 antagonists}

Mepolizumab: Mepolizumab is a humanized anti-IL-5 antagonist antibody, approved in November 2015 as an addon subcutaneous therapy for patients older than 12 years with severe eosinophilic asthma. IL-5 cytokines regulate the growth, recruitment, activation, and life cycle of eosinophils. Mepolizumab binds to IL-5 and inhibit IL-5 signaling, thereby reducing the production and survival of eosinophils [4,8-11].

Mepolizumab is administered as a $100 \mathrm{mg}$ subcutaneous injection every 4 weeks in the upper arm, thigh, or abdomen in stable patients diagnosed with severe eosinophilic 
asthma, but not during an acute asthma exacerbation, acute bronchospasm, or status asthmaticus. Varicella vaccination should be considered prior to initiating mepolizumab treatment, due to two reported serious herpes zoster adverse events in clinical trials. Headache (19\%), back pain (5\%), and injection site reactions (8\%), was reported in all mepolizumab clinical trials. Mepolizumab should be prescribed cautiously to patients older than 65 years with severe asthma due to a higher potential for increased sensitivity. There are insufficient data for using mepolizumab in pregnancy, lactation, or a pediatric population less than 12 years of age $[2,4,11,17]$.

The DREAM study was the largest study conducted to establish the safety and efficacy of different doses of mepolizumab versus placebo. More than 600 patients with severe asthma were enrolled in this multicentre, double blinded, placebo-controlled study for 52 weeks, to establish the safety and efficacy of mepolizumab $75 \mathrm{mg}, 250 \mathrm{mg}$, and $750 \mathrm{mg}$ intravenously compared to placebo. The rate of a clinically significant exacerbation per patient per years was reduced by 48\% (95\% CI $31-61 \%$, P < 0.0001) in Mepolizumab 75mg, and 39\% (95\% CI $19-54 \%, \mathrm{P}=0.0005$ ) in Mepolizumab 250mg, and with 52\% (95\% CI 36-64\%, P < 0.0001 ) in mepolizumab $750 \mathrm{mg}$ compared to placebo. The geometric mean of blood eosinophils was reduced at week 52 in subjects given mepolizumab $75 \mathrm{mg}(0.22$ 95\% CI 0.0 .18 - 0.27, $\mathrm{P}<0.0001)$, and in mepolizumab $250 \mathrm{mg}(0.1495 \% \mathrm{CI}$; $0.12-0.18, \mathrm{P}<0.0001)$, and in mepolizumab 750mg $(0.1295 \%$ CI $0.09-0.14, \mathrm{P}<0.0001)$. Emergency department visits and hospital admissions were also reduced in the mepolizumab group compared to placebo, however mepolizumab did not have a significant clinical or statistical effect on the pulmonary function test (FEV1), or AQLQ scores. Mepolizumab was well tolerated by the participants with no serious side effects described in both groups, but infusion related adverse events were noted in the mepolizumab group compared to placebo [18].

Ortega, et al. in the Mensa trial, a randomized double blind, double dummy confirmatory trial, included 567 patients with severe asthma, and recurrent exacerbation despite a high dose of ICS. Patients were randomized to three groups of mepolizumab $75 \mathrm{mg}$ intravenously, or mepolizumab $100 \mathrm{mg}$ subcutaneously, or placebo for 32 weeks. The rate of asthma exacerbation was significantly reduced by 53\% (95\% CI; 36 - 65, $\mathrm{P}<0.001$ ) in patients who received the subcutaneous mepolizumab, and by $47 \%$ (95\% CI; $28-60, \mathrm{P}<0.001)$ in the intravenous mepolizumab group compared to the placebo group. The need for hospitalization or emergency visit was reduced by $61 \%$ in the subcutaneous mepolizumab group, and by $32 \%$ in intravenous mepolizumab group compared to placebo. The 5-item Asthma Control Questionnaire (ACQ-5), and the George's Respiratory Questionnaire (SGRQ) scores were not significantly different in both mepolizumab groups compared to placebo. The mean increase from baseline FEV1 was not significantly improved between both mepolizumab groups and the placebo group. In addition, the safety profile was similar among all groups [19].

Bel, et al., investigated the sparing effect of mepolizumab $100 \mathrm{mg}$ subcutaneous of oral glucocorticoids in steroid dependent severe asthma patients in a randomized doubleblind trial, the SIRIUS trial, for 20 weeks. It was confirmed that the reduction in the daily dose of glucocorticoids in the mepolizumab group was higher and significant different compared to the placebo group (OR 2.39; 95\% CI; 1.25-4.56). The daily oral glucocorticoids dose was reduced by $90 \%$ to $100 \%$ in $23 \%$ of the patients in the mepolizumab group compared to the placebo group (11\%), and a reduction of $70 \%$ to less than $90 \%$ in $17 \%$ of the mepolizumab group patients compared to $8 \%$ in the placebo group. In addition to oral the glucocorticoids dose reduction, asthma exacerbation and overall improvement in asthma control was confirmed in SIRIUS trial. In a post-hoc analysis of both the MENSA and SIRIUS trials, an found an overall reduction in asthma exacerbation of $52 \%$ in patients with an eosinophil count of 150 cells $/ \mu \mathrm{l}$ and $70 \%$ in patients with eosinophil count of 500 cells/ $\mu$ l. The significant reduction in asthma exacerbation is highly predicted in patients with an eosinophils count of more than 150 cells $/ \mu \mathrm{l}[20]$.

In a recent study conducted by Chupp, et al. (MUSCA), mepolizumab was associated with a significant improvement in HRQOL in patients with severe eosinophilic asthma. The SGRO total score improved from baseline compared to 24 weeks of treatment by -7.7 ( $95 \%$ CI -10.5 to $-4.9, \mathrm{P}<0.0001)$. Overall, mepolizumab significantly reduced the rate of asthma exacerbation in patients with severe eosinophilic asthma and had a good oral glucocorticoids dose sparing effect, with a tolerable safety profile. However, it failed to show a statistically significant improvement in pulmonary function [21].

COSMOS was a 52 weeks open label extension phase IIIb study where mepolizumab $100 \mathrm{mg}$ subcutaneously was offered to all previously enrolled patients in both the MENSA and SIRIUS studies regardless their initial allocation in the trials. In COSMOS, mepolizumab's long term safety (adverse events and serious adverse events), in addition to efficacy (annual asthma exacerbation rate, and oral glucocorticoids use) were evaluated. The prevalence of adverse and serious adverse events was low compared to previous trials, with $<1 \%$ of the patients experiencing cerebrovascular or cardiovascular side events (stroke, deep vein thrombosis, atrial fibrillation, and myocardial infarction). The reported adverse events in the trail was similar to those in previous trials in the treatment and placebo groups including nasopharyngitis (30\%), upper respiratory tract infection (16\%), and worsening asthma symptoms (14\%). The exacerbation rate as well as hospitalization and emergency department visits showed a low prevalence rate which is consistent with previous studies. Mepolizumab efficacy was noticed to be durable with a stable response even after 84 weeks of treatment [22]. 
COLUMBA was an open label, long term extension safety study of mepolizumab in asthmatic patients. All previously enrolled patients with severe eosinophilic asthma in the DREAM study who received mepolizumab $100 \mathrm{mg}$ subcutaneous every 4 weeks and followed up according to the protocol, had been re-evaluated for a total duration of a maximum of 4.5 years (average 3.5 years). Frequently reported adverse events while on treatment were respiratory tract infection (67\%), headache (29\%), bronchitis (21\%), asthma worsening (27\%), and injection site reaction (3\%). Serious side events were reported in $23 \%$ of the patients. Six patients died with one death related to mepolizumab [23]. The Saudi Initiative for Asthma (SINA 2019) stated that all IL-5 antibody including mepolizumab should be continued for 6-12 months. The guidelines was published before the COLUMBA study release and the duration of mepolizumab use might be exempted from the general role for IL- 5 antibodies $[2,23,24]$.

Reslizumab: Resilzumab is an anti-IL-5 antibody that neutralizes circulating IL- 5 and inhibits its bioactivity related to eosinophils. It was approved by the US FDA in March 2016, as an add-on maintenance treatment for patients 18 years or older with severe eosinophilic asthma. The approved dose is 3 $\mathrm{mg} / \mathrm{kg}$ as an intravenous infusion over 20-50 minutes every 4 weeks [4,8-11,25-27].

Castro, et al. in their randomized, double-blind, placebocontrolled study conducted in 2011, using 53 patients with severe eosinophilic asthma who were not controlled on a high dose of ICS, report a significant reduction in sputum and blood eosinophils $(95.45 \%$ vs. $38.75 \%$ in placebo group, $\mathrm{P}=$ 0.0068). The effect of resilzumab on lung function compared to placebo showed a statistically significant improvement from the baseline FEV1, percentage of predicted FEV1, and FVC. In addition, the ACQ score improved in 59\% of patients in the resilzumab group compared to $40 \%$ in the placebo group by 0.5 (OR $2.06(95 \% \mathrm{CI} ; 0.88-4.86) \mathrm{P}=0.0973)$, considered as a minimal clinically significant improvement [28].

Castro, et al. in their duplicated, multicenter, double-blind, parallel-group, randomized, placebo-controlled phase III trial, enrolled 953 patients randomly assigned to two groups. The frequency of asthma exacerbation in the resilzumab group was significantly reduced compared to the placebo group after more than 52 weeks of treatment. In addition, the time to the first asthma exacerbation was prolonged significantly in the resilzumab group. Two cases of anaphylactic reaction which responded to standard treatment were reported in the resilzumab group compared to the placebo [29].

To determine whether the baseline serum eosinophils influenced the efficacy outcome of reslizumab, two randomized clinical trials were conducted. Corren, et al. randomly assigned 492 patients with severe poorly controlled asthma and blood eosinophils $<400$ cells $/ \mu$ lo a reslizumab and placebo group. The aim of the study was to measure the change in FEV1 from baseline to week 16, improvement of asthma symptoms measured with the ACQ-7, the reduction of rescue medication use as well as the safety profile. No statistically significant effect was observed in terms of the FEV1 in the reslizumab group with eosinophils $<400$ cells/ $\mu \mathrm{l}$ compared to placebo, and a modest effect was reported with the ACQ-7 score $(P=0.0457)$. Overall reslizumab was well tolerated with no significant adverse events among the groups [30]. In contrast, Bjermer, et al. randomly assigned 315 patients with severe uncontrolled asthma on a medium dose ICS, with blood eosinophils $>400$ cells $/ \mu$ lo a reslizumab $(0.3 \mathrm{mg} / \mathrm{kg}$ and $3 \mathrm{mg} / \mathrm{kg})$ and placebo group. Lung function (FEV1), ACQ and AQLQ scores were significantly improved in resilzumab $(3 \mathrm{mg} / \mathrm{kg}$ ) group compared to placebo, and the adverse events were similar to previous studies [31]. These two trials (Corren, et al. l and Bjermer, et al.) demonstrated that reslizumab is more efficacious in patients with severe eosinophilic asthma (blood eosinophil $>400$ cells $/ \mu l$ ) $[30,31]$. Brusselle, et al. in their 2017 study, assessed the efficacy of reslizumab in patients receiving baseline treatment per GINA step 4 and 5 guidelines, and found that reslizumab reduced the exacerbation rate by $53 \%(95 \% \mathrm{CI} 0.36-0.62)$ and $72 \%(95 \%$ CI $0.15-0.52$ ) in step 4 and step 5 groups respectively [32]. Several studies reported that because of the weight- based dosing strategy for reslizumab, it was more effective than a fixed dose mepolizumab in reducing sputum eosinophils and other asthma control outcomes $[23,26]$.

Benralizumab: Benralizumab is a humanized afucosylated monoclonal antibody directed against the alpha subunit of IL-5 receptors (IL5R $\alpha$ ), which induces direct and nearly completed depletion of eosinophils by means of natural killer cell-mediated antibody dependent cellular cytotoxic effects and apoptosis. Benralizumab was approved in November 2017 by the US FDA for patients with severe asthma older than 12 years. The approved dose is $30 \mathrm{mg}$ subcutaneously every 4 weeks for 3 doses, then every 8 weeks [4,8-11,33-35].

Several phase II and phase III studies were conducted to assess the safety and efficacy of benralizumab in adult patients with severe eosinophilic asthma while on oral corticosteroid or high dose of ICS/LABA. The SIROCCO trail, a randomized, double-blind, parallel-group, placebocontrolled trial conducted over 48 weeks to assess the efficacy of different benralizumab regimens (30mg every 4 weeks, and $30 \mathrm{mg}$ every 8 weeks) versus placebo for 48 weeks. The annual asthma exacerbation rate was significantly reduced in both benralizumab groups (Q4W 0.55, 95\% CI 0,42 - 0,71, $\mathrm{P}<0.0001$, and Q8W 0.49, 95\% CI $0.37-0.64, \mathrm{P}<0.0001$ ) compared to placebo. With both regimens in the benralizumab group, prebronchiodialtor FEV1 significantly improved over 48 weeks compared to the placebo group. The authors reported that asthma symptoms worsening (13\%), and nasopharyngitis $(12 \%)$ were the most common adverse events 
in both groups [36]. In the CALIMA trial, the investigators evaluated the efficacy and safety of benralizumab as an addon therapy for patients with severe uncontrolled asthma using the same regimen as in the SIROCCO trial but for a longer duration (56 weeks). The results from the CALIMA trial confirmed the previous studies, however nasopharyngitis was reported in $21 \%$ of the patients in the benralizumab group compared to placebo [37]. The oral glucocorticoids sparing effect of benralizumab was evaluated in the ZONDA trial, a 28-week randomized, placebo-controlled trial conducted with 145 patients, diagnosed with severe eosinophilic asthma on oral corticosteroid. The participants were randomized to a benralizumab $30 \mathrm{mg}$ subcutaneous every 4 weeks or every 8 weeks and a placebo group. The annual exacerbation rate, asthma symptoms, lung function and safety were assessed. Oral glucocorticoids use was reduced by $75 \%$ in patients receiving either of the benralizumab regimens compared to the placebo (25\%, $\mathrm{P}<0.001$ in both comparisons). The Q8W benralizumab group showed an annual asthma exacerbation reduction of $70 \%$ compared to placebo ( 0.54 vs. $1.83, \mathrm{P}=0.003)$. Pulmonary function was not significantly affected at 28 week duration and there was no statistical difference between all the groups. The reported adverse effect was the same in all the groups with worsening asthma symptoms (13\%), nasopharyngitis (17\%) and bronchitis (13\%). The non-significant effect on the lung function or asthma symptoms might be related to the short duration of this study [38]. Benralizumab efficacy and safety was studied in mild-moderate persistent asthma (BISE trial), with patients using a low to medium dose of ICS. The participants were randomised to benralizumab $30 \mathrm{mg}$ subcutaneous every 4 weeks or placebo for 12 weeks. The effect on pulmonary function was not statistically significant between the study groups, indicating that benralizumab use is not defensible in this group of patients, however depleted blood eosinophils were observed in the benralizumab group [39].

Long-term safety and efficacy were evaluated in a randomized, multicenter, double-blind, parallel-group, phase III extension study for the CALIMA and SIROCCO trials for 56 weeks for adults and 108 weeks for adolescents (BORA trial) [40]. Patients were maintained on benralizumab $30 \mathrm{mg}$ ever 4 weeks and every 8 weeks. The most frequent reported adverse events were viral upper respiratory tract infection (15\%), worsening of asthma symptoms (3\%), and injection site reaction (2\%), without any report related to serious helminthic infection or anaphylactic reaction. Serious adverse events were reported in $10-11 \%$ of the participants of the BORA trials compared to the CALIMA and SIROCCO trials $(13 \%$ and $9 \%)$. The relative similarity in reporting serious adverse events between BORA and CALIMA, and SIROCCO, may be due to the investigators not being blinded to the drug (BORA trial) which may have biased their reporting of serious adverse events in relation to benralizumab [40]. Overall benralizumab proved to be safe and effective over two years duration, without any consequence of eosinophil depletion, or evidence of opportunistic infections [41]. However, clinician should avoid prescribing benralizumab or any other anti-IL-5 if helminthic infection is present as per SINA-2019 guidelines1. The WINDWARD program and three phase III trials (ANDHI, MIRACLE and SOLANA) are on-going, as well as the approval process at the US FDA for intravenous benralizumab [41].

\section{IL-4 antagonists}

Dupilumab: Dupilumab is a human monoclonal antibody to the IL-4 receptor alpha subunit (IL4R $\alpha$ ). It inhibits binding and downstream signalling in both IL-4 and IL-13, two important cytokines in developing Th2 and IgE producing B-cells. It has recently been approved by the US FDA to treat patients older than 12 years with moderate-severe eosinophilic asthma with blood eosinophils more than 300 cells/ $\mu \mathrm{l}$ as well as oral steroid dependent severe asthma. Dupilumab is administered in a dose of $400 \mathrm{mg}$ followed by $200 \mathrm{mg}$ subcutaneous every 2 weeks for patients with the eosinophilic phenotype asthma, for oral steroid dependent patients the dose is $600 \mathrm{mg}$ followed by $300 \mathrm{mg}$ subcutaneous every two weeks $[2,4,8-11]$.

The efficacy of dupilmuab in terms of the asthma exacerbation recurrence rate was confirmed in a phase IIa trial [41]. Patients with severe persistent eosinophilic asthma, using a medium to high dose of ICS/LABA, were randomized to receive dupilmuab $300 \mathrm{mg}$ subcutaneously once weekly or placebo. Asthma exacerbation was reduced by $87 \%$ in the dupilmuab group, with a significant improvement in asthma symptoms and lung functions compared to placebo [42]. The same investigators [41], conducted arandomized,double-blind, placebo-controlled, parallel group, phase IIb trial, and after three years, they confirmed their initial findings of reducing the annual exacerbation rate with dupilmuab administered every two weeks compared to placebo, regardless the baseline eosinophil level. Overall the asthma symptoms as measured through the ACQ-5 showed an improvement in the total scores at week 24, in the group receiving dupilmuab every 2 weeks compared to duplimuab every 4 weeks and placebo. The FEV1 was significantly increased from baseline to week 12 (0.35L to $0.43 \mathrm{~L}$ ), and sustained through the 24 -week treatment period. In addition, dupilmuab decreased the biomarkers associated with Th2 inflammation (FENO, eotaxin-3, TARC), and serum IgE. The reported adverse events among all groups were upper respiratory tract infection, headache and injection site reaction with no significant difference between groups [43]. In a recent trial, published in June 2018 by Rabe, et al. the effectiveness of dupilmuab in reducing the oral glucocorticoid dose in patients with severe asthma was investigated. In this randomized, double blind, placebo-controlled trial, the patients were assigned to either dupilmuab $300 \mathrm{mg}$ every 2 weeks for 24 weeks or a placebo group [44]. The oral glucocorticoid dose was totally discontinued in $48 \%$ of the dupilmuab group compared to $25 \%$ of the placebo group. The majority $(80 \%)$ of the dupilmuab group reduced the total dose with $50 \%$ versus the placebo group (50\%). The oral glucocorticoid dose was 
reduced to less than $5 \mathrm{mg}$ per day in $69 \%$ of the duplimuab group compared to $33 \%$ in placebo group. The other outcomes including the improved rate of exacerbation, improved lung function, safety profile, and transient increase in eosinophil level was confirmed in this trial $[44,45]$.

It is noteworthy that inhibiting IL-4 signaling will inhibit IL-13 signaling as it highlights the fact that the activation of IL13 causes the release of different biomarkers including FENO, and blood periostin. These biomarkers could be used in future to monitor the effectiveness of dupilmuab since it shares the same activation axis as IL-13 [16].

\section{Conclusion}

Asthma is defined as a heterogeneous disease that affects up to $18 \%$ of the Saudi population, where approximately $20 \%$ of patients do not achieve control that lead to increased mortality, morbidity and overall healthcare cost. Different targets had been developed to treat patients with severe asthma who are not responsive to ICS, including IgE, IL$4 / 13$, and IL-5, IL-17, TSLP, in addition to many others being investigated. All biologic therapy for severe asthma should be initiated and monitored by specialists due to many challenges in initiating and monitoring the therapy. Severe asthma exacerbation is one of the challenges facing the clinicians when discontinuing IgE antagonist after long term therapy. IL-5 antagonists are promising in treating severe asthma with acceptable safety and efficacy profile; however, clinician should avoid prescribing them if any helminthic infection is present as per SINA-2019 guidelines. Different monitoring parameters could be used to guide the therapy with IL-4 antagonists (FENO, and blood periostin), in the future since inhibiting IL-4 pathway will cause inhibition in IL-13 signaling and as a result it will lead to decrease in the release of different biomarkers.

\section{Acknowledgment}

1. Acknowledgment for Prof. Mohamed Al Gobain (Pulmonary Consultant at Ministry of the National Guard Health Affairs, Riyadh, Saudi Arabia) for providing guidance and review of the manuscript.

2. Acknowledgment for Dr. Susanna Wright from King Abdullah International Medical Research Center, Riyadh, Saudi Arabia, publication office for her contribution in language editing of the manuscript.

Article was sponsored by King Abdullah International Medical Research center, Saudi Arabia-Riyadh.

\section{References}

1. Asthma GI. Global Strategy for Asthma Management and Prevention: GINA; 2018.

2. Al-Moamary MS, Alhaider SA, Alangari AA, Al Ghobain MO, Zeitouni MO, et al. The Saudi Initiative for Asthma-2019 Update: Guidelines for the diagnosis and management of asthma in adults and children. Ann Thorac Med. 2019; 14: 3-48.

PubMed: https://www.ncbi.nlm.nih.gov/pubmed/30745934
3. McCracken J, Veeranki SP, Ameredes BT, Calhoun WJ. Diagnosis and Management of Asthma in Adults: A Review. JAMA. 2017; 318: 279-290. PubMed: https://www.ncbi.nlm.nih.gov/pubmed/28719697

4. McCracken J, Tripple JW, Calhoun WJ. Biologic Therapy in the Management of Asthma. Curr Opin Allergy Clin Immunol. 2016; 16 : 375-382.

PubMed: https://www.ncbi.nlm.nih.gov/pubmed/27362324

5. Al Ghobain MO, Algazlan SS, Oreibi TM. Asthma prevalence among adults in Saudi Arabia. Saudi Med J. 2018; 39: 179-184.

PubMed: https://www.ncbi.nlm.nih.gov/pubmed/29436567

6. Stephen T Holgate, Riccardo Polosa. The mechanisms, diagnosis, and management of severe asthma in adults. Lancet. 2006; 368: 780-793. PubMed: https://www.ncbi.nlm.nih.gov/pubmed/16935689

7. Canonica GW, Senna G, Mitchell PD, O'Byrne PM, Passalacqua G, et al. Therapeutic interventions in severe asthma. World Allergy Organ J. 2016; 9: 1-12.

PubMed: https://www.ncbi.nlm.nih.gov/pubmed/27942351

8. Yang X, Feng HR, Chen ZM, Ying SM. Biologic Targeting: New and Effective Therapeutic Approaches against Severe Asthma. Chin Med J. 2018; 31: 1009-1012.

PubMed: https://www.ncbi.nlm.nih.gov/pmc/articles/PMC5937305/

9. Diver S, Russell RJ, Brightling CE. New and emerging drug treatments for severe asthma. Clin Exp Allergy. 2018; 48: 241-252.

PubMed: https://www.ncbi.nlm.nih.gov/pubmed/29315966

10. Linda Zhu, Ciaccio CE, Casale TB. Potential new targets for drug development in severe asthma. World Allergy Organ J. 2018; 11: 1-9. PubMed: https://www.ncbi.nlm.nih.gov/pubmed/30386455

11. Patel TR, Sanjiv Sur. Ige and eosinophils as therapeutic targets in asthma. Curr Opin Allergy Clin Immunol. 2017; 17: 42-49.

PubMed: https://www.ncbi.nlm.nih.gov/pubmed/27906698

12. Hanania NA, Alpan O, Hamilos DL, Condemi JJ, Reyes-Rivera I, et al. Omalizumab in Severe Allergic Asthma Inadequately Controlled with Standard Therapy. Ann Intern Med. 2011; 154: 573-582. PubMed: https://www.ncbi.nlm.nih.gov/pubmed/21536936

13. Humbert M, Beasley R, Ayres J, Slavin R, Hébert J, et al. Benefits of omalizumab as add-on therapy in patients with severe persistent asthma who are inadequately controlled despite best available therapy (GINA 2002 step 4 treatment): INNOVATE. Allergy. 2005; 60: 309-316. PubMed: https://www.ncbi.nlm.nih.gov/pubmed/15679715

14. Braunstahl GJ, Chen CW, Maykut R, Georgiou P, Peachey G, et al. The eXpeRience registry: The 'real-world 'effectiveness of omalizumab in allergic asthma. Respir Med. 2013; 107: 1141-1151.

PubMed: https://www.ncbi.nlm.nih.gov/pubmed/23721684

15. Iribarren C, Rahmaoui A, Long AA, Szefler SJ, Bradley MS, et al. Cardiovascular and cerebrovascular events among patients receiving omalizumab: Results from EXCELS, a prospective cohort study in moderatetosevereasthma.JAllergyClin Immunol.2017;139:1489-1495. PubMed: https://www.ncbi.nlm.nih.gov/pubmed/27639934

16. Caruso M, Morjaria J, Emma R, Amaradio MD, Polosa R. Biologic agents for severe asthma patients: clinical perspectives and implications. Intern Emerg Med. 2018; 13: 155-176.

PubMed: https://www.ncbi.nlm.nih.gov/pubmed/29238905

17. Menzella F, Lusuardi M, Montanari G, Galeone C, Facciolongo N, et al. Clinical usefulness of mepolizumab in severe eosinophilic asthma. Ther Clin Risk Manag. 2016; 12: 907-916.

PubMed: https://www.ncbi.nlm.nih.gov/pubmed/27354806

18. Pavord ID, Korn S, Howarth P, Bleecker ER, Buhl R, et al. Mepolizumab for severe eosinophilic asthma (DREAM): a multicenter, double-blind, placebo-controlled trial. Lancet. 2012; 380: 651-659. PubMed: https://www.ncbi.nlm.nih.gov/pubmed/22901886 
19. Ortega HG, Liu MC, Pavord ID, Brusselle GG, FitzGerald JM, et al Mepolizumab Treatment in Patients with Severe Eosinophilic Asthma. N Engl J Med. 2014; 371: 1198-1207.

PubMed: https://www.ncbi.nlm.nih.gov/pubmed/25199059

20. Elisabeth HB, Wenzel SE, Thompson PJ, Prazma CM, Keene ON, et al. Oral Glucocorticoid-Sparing Effect of Mepolizumab in Eosinophilic Asthma. N Engl J Med. 2014; 371: 1189-1197.

PubMed: https://www.ncbi.nlm.nih.gov/pubmed/25199060

21. Chupp GL, Bradford ES, Albers FC, Bratton DJ, Wang-Jairaj J, et al. Efficacy of mepolizumab add-on therapy on health-related quality of life and markers of asthma control in severe eosinophilic asthma (MUSCA): a randomised, double-blind, placebo-controlled parallel-group, multicentre, phase 3b trial. Lancet. 2017; 5: 390-400. PubMed: https://www.ncbi.nlm.nih.gov/pubmed/28395936

22. Lugogo N, Domingo C, Chanez P, Leigh R, Gilson MJ, et al. Long-term efficacy and safety of Mepolizumab in patients with severe eosinophilic Asthma: A Multi-center, Open-label, Phase IIIb Study. Clin Ther. 2016; 38: 2058-2069.

PubMed: https://www.ncbi.nlm.nih.gov/pubmed/27553751

23. Khatri S, Moore W, Gibson PG, Leigh R, Bourdin A, et al. Assessment of the long-term safety of mepolizumab and durability of clinical response in patients with severe eosinophilic asthma. J Allergy Clin Immunol. 2019; 143: 1742-1751.

PubMed: https://www.ncbi.nlm.nih.gov/pubmed/30359681

24. Emma R, Morjaria JB, Fuochi V, Polosa R, Caruso M. Mepolizumab in the management of severe eosinophilic asthma in adults: current evidence and practical experience. Ther Adv Respir Dis. 2018; 12:1-12. PubMed: https://www.ncbi.nlm.nih.gov/pubmed/30354852

25. Galo AP, Labor M, Tiotiu A, Baiardini I, Scichilone N, et al. Impact of reslizumab on outcomes of severe asthmatic patients: current perspectives. Patient Relat Outcome Meas. 2018; 9: 267-273. PubMed: https://www.ncbi.nlm.nih.gov/pmc/articles/PMC6103306/

26. Castro M, Mathur S, Hargreave F, Boulet LP, Xie F, et al. Reslizumab for poorly controlled eosinophilic asthma: a randomized, placebocontrolled study. Am J Respir Crit Care Med 2011; 184: 1125-1132. PubMed: https://www.ncbi.nlm.nih.gov/pubmed/21852542

27. Castro M, Zangrilli J, Wechsler ME, Bateman ED, Brusselle GG, et al. Reslizumab for inadequately controlled asthma with elevated blood eosinophil counts: results from two multicentre, parallel, double-blind randomised, placebo-controlled, phase 3 trials. Lancet Respir Med. 2015; 3: 355-366.

PubMed: https://www.ncbi.nlm.nih.gov/pubmed/25736990

28. Corren J, Weinstein S, Janka L, Zangrilli J, Garin M. Phase 3 study of randomized in patients with poorly controlled asthma: effect across a board range of eosinophil counts. Chest. 2016; 150: 799-810. PubMed: https://www.ncbi.nlm.nih.gov/pubmed/27018175

29. Bjermer L, Lemiere C, Maspero J, Weiss S, Zangrilli J, et al. Reslizumab for inadequately controlled asthma with elevated blood eosinophi levels: a randomized phase 3 study. Chest. 2016; 150: 789-798. PubMed: https://www.ncbi.nlm.nih.gov/pubmed/27056586

30. Brusselle G, Canvin J, Weiss S, Sun SX, Buhl R. Stratification of eosinophilic asthma patients treated with reslizumab and GINA Step 4 or 5 therapy. ERJ Open Res. 2017; 3: 1-9.

PubMed: https://www.ncbi.nlm.nih.gov/pubmed/28845430

31. Pelaia C, Vatrella A, Bruni A, Terracciano R, Pelaia G. Benralizumab in the treatment of severe asthma: design, development and potential place in therapy. Drug Des Devel Ther. 2018; 12: 619-628. PubMed: https://www.ncbi.nlm.nih.gov/pubmed/29606855

32. Pelaia C, Calabrese C, Vatrella A, Busceti MT, Garofalo E, et al. Benralizumab: From the Basic Mechanism of Action to the Potential Use in the Biological Therapy of Severe Eosinophilic Asthma. Biomed
Res Int. 2018; 2018: 1-9.

PubMed: https://www.ncbi.nlm.nih.gov/pubmed/29862274

33. Menzella F, Lusuardi M, Galeone C, Facciolongo N, Zucchi L. The clinical profile of benralizumab in the management of severe eosinophilic asthma. Ther Adv Respir Dis. 2016; 10: 534-548.

PubMed: https://www.ncbi.nlm.nih.gov/pubmed/27612492

34. Bleecker ER, FitzGerald JM, Chanez P, Papi A, Weinstein SF, et al. Efficacy and safety of benralizumab for patients with severe asthma uncontrolled with high-dosage inhaled corticosteroids and long-acting $\beta 2$-agonists (SIROCCO): a randomised, multicentre, placebo-controlled phase 3 trial. Lancet. 2016; 388: 2115-2127.

PubMed: https://www.ncbi.nlm.nih.gov/pubmed/27609408

35. FitzGerald JM, Bleecker ER, Nair P, Korn S, Ohta K, et al. Benralizumab, an anti-interleukin-5 receptor a monoclonal antibody, as add-on treatment for patients with severe, uncontrolled, eosinophilic asthma (CALIMA): a randomised, double-blind, placebo-controlled phase 3 trial. Lancet. 2016; 388: 2128-2141.

PubMed: https://www.ncbi.nlm.nih.gov/pubmed/27609406

36. Nair N. Oral Glucocorticoid-Sparing Effect of Benralizumab in Severe Asthma. N Engl J Med. 2017; 376: 2448-2458.

37. Ferguson GT, FitzGerald JM, Bleecker ER, Laviolette M, Bernstein D, et al. Benralizumab for patients with mild to moderate, persistent asthma (BISE): a randomised, double-blind, placebo-controlled, phase 3 trial. Lancet Respir Med. 2017; 5: 568-576.

PubMed: https://www.ncbi.nlm.nih.gov/pubmed/28545978

38. Busse WW, Bleecker ER, FitzGerald JM, Ferguson GT, Barker P, et al Long-term safety and efficacy of benralizumab in patients with severe, uncontrolled asthma: 1-year results from BORA phase 3 extension trial. Lancet. 2019; 7: 46-59.

PubMed: https://www.ncbi.nlm.nih.gov/pubmed/30416083

39. Bourdin A, Husereau D, Molinari N, Golam S, Siddiqui MK, et al. Matchingadjusted indirect comparison of benralizumab versus interleukin-5 inhibitors for the treatment of severe asthma: a systematic review. Eur Respir J. 2018; 52: 1-13

PubMed: https://www.ncbi.nlm.nih.gov/pubmed/30309978

40. Wenzel S, Ford L, Pearlman D, Spector S, Sher L, et al. Dupilumab in persistent asthma with elevated eosinophil levels. N Engl J Med. 2013; 368: $2455-2466$

PubMed: https://www.ncbi.nlm.nih.gov/pubmed/23688323

41. Wenzel S, Castro M, Corren J, Maspero J, Wang L, et al. Dupilumab efficacy and safety in adults with uncontrolled persistent asthma despite use of medium-to-high-dose inhaled corticosteroids plus a long-acting $\beta 2$ agonist: a randomised double-blind placebo-controlled pivotal phase $2 \mathrm{~b}$ dose-ranging trial. Lancet. 2016; 388: 31-44. PubMed: https://www.ncbi.nlm.nih.gov/pubmed/27130691

42. Rabe KF. Efficacy and Safety of Dupilumab in Glucocorticoid-Dependent Severe Asthma. N Engl J Med. 2018; 378: 2475-2485.

43. Menzella F, Galeone C, Bertolini F, Castagnetti C, Facciolongo N Innovative treatments for severe refractory asthma: how to choose the right option for the right patient? J Asthma Allergy. 2017; 10: 237-247. PubMed: https://www.ncbi.nlm.nih.gov/pubmed/28919788

44. Pelaia G, Vatrella A, Busceti MT, Gallelli L, Preianò M, et al. Role of biologics in severe eosinophilic asthma focus on reslizumab. Ther Clin Risk Manag. 2016; 12: 1075-1082.

PubMed: https://www.ncbi.nlm.nih.gov/pubmed/27445482

45. Sahota J, Robinson DS. Update on new biologics for intractable eosinophilic asthma: impact of reslizumab. Drug Des Devel Ther. 2018; 12: 1173-1181.

PubMed: https://www.ncbi.nlm.nih.gov/pubmed/29780238 